\title{
La Psicología Educativa en España. Unas Notas Históricas sobre sus Primeros Pasos
}

\section{Educational Psychology in Spain. Historical Notes on its Early Years}

\author{
Helio Carpintero \\ UDIMA-Universidad a Distancia de Madrid, España
}

\begin{abstract}
Resumen. La renovación de la sociedad española, desde finales del siglo XIX, tuvo uno de sus pilares básicos en la modernización y fortalecimiento de las instituciones educativas, a través de las cuales los distintos grupos socialmente activos buscaron lograr una regeneración social. El interés por la psicología como disciplina que sirva de base a la teoría y la práctica educativa adquiere fuerza en España a finales del siglo XIX y comienzos del siglo XX, especialmente por obra de educadores pertenecientes a la tradición intelectual del Krausismo y de la Institución Libre de Enseñanza. En esta línea se presta atención a algunas contribuciones especializadas, de González Serrano, Saiz Amor, Simarro, Barnés, Viqueira y Rodriguez Lafora. También se tienen en cuenta otras tradiciones más fuertemente vinculadas a condicionamientos ideológicos y políticos. Así, se recuerda la aportación de la Escuela Moderna de Ferrer Guardia, inspirada en principios anarquistas y librepensadores, así como las de otros educadores inspirados en la doctrina católica, como Manjón y Barbens.

Palabras clave: historia de la psicología educativa en España, movimiento krausista, Institución Libre de Enseñanza.
\end{abstract}

\begin{abstract}
The renewal that took place in Spanish society from the late nineteenth century onwards had as one of its cornerstones the modernization and strengthening of educational institutions. Indeed, it was through these institutions that different groups active in society sought to bring about social regeneration. Interest in psychology as a discipline that forms the basis of the educational theory and practice gained momentum in Spain in the late nineteenth and early twentieth century, especially through the work of teachers belonging to the Krausism intellectual tradition and the Institutión Libre de Enseñanza. In this sense, the contributions of certain specialists are particularly noted: González Serrano, Saiz Amor, Simarro, Barnés, Viqueira and Rodriguez Lafora. Other traditions are also taken into account, more politically and ideologically conditioned. In this way, the contribution is recalled of Ferrer Guardia's Modern School, inspired by anarchist and freethinker principles, as well as other inspired educators from within the Catholic doctrine, such as Manjón and Barbens.

Keywords: history of educational psychology in Spain, Krausism movement, Institución Libre de Enseñanza.
\end{abstract}

En la tradición de la psicología española, hay una veta continuada por la que discurren las reflexiones y estudios sobre la realidad educativa en sus diversos

La correspondencia sobre el artículo debe enviarse al autor al email: heliodoro.carpintero@udima.es aspectos -teorías, escuelas, variables psicológicas relevantes en el fenómeno de la educación...- . De esta suerte, su evolución corre paralela a la de la historia general, y sus fluctuaciones evidencian las diferentes peripecias por que ha pasado este campo científico y profesional a lo largo de ya casi un siglo y medio. 
Al lanzar la mirada hacia el futuro, buscando un horizonte de posibilidades y nuevos desarrollos, tiene sentido volverla también hacia el pasado, para cobrar conciencia de la trayectoria que hemos recorrido. Unamuno decía que la vuelta sobre el pasado era la necesaria preparación para dar un salto hacia el futuro. Es cierto que nuestro tiempo contempla un rapidísimo cambio de circunstancias, de capacidades técnicas, e incluso de situaciones concretas en que interaccionan los individuos. El mundo de las realidades virtuales ha transformado la vida contemporánea, y por ello mismo, también la educación. Muchas cuestiones que son hoy problema son tales que no se pudo pensar antes en ellas porque ni siquiera cabía imaginar las condiciones de su aparición. Pero, por otro lado, la realidad misma de la educación, en un sentido profundo, conserva en medio de grandes cambios la estructura básica de la relación maestro- discípulo, y aquella otra que relaciona el mundo presente con otro futuro, a cuyas líneas ideales se procura orientar los espíritus que se educa, poniendo en juego las variables psicológicas que modulan la acción. Esta permanencia de las dimensiones del proceso educativo, por debajo de sus innumerables variaciones en la historia reciente, hace que la mirada al pasado pueda todavía ser útil como fuente de inspiración para nuevos procedimientos aplicables a nuestra labor. No todo es vino viejo en odres nuevos; pero tampoco es eficaz el adanismo, que cree cada día estar estrenando el mundo por primera vez. La historia es maestra de la vida, aunque en ocasiones cueste entender lo que está diciendo en lenguas que a veces no nos resultan plenamente inteligibles.

Cuando se contempla lo que ha sido el campo de la psicología educativa en nuestro país, durante las primeras décadas del siglo XX, cuando se iba configurando y definiendo como un espacio a la vez científico y profesional, dos rasgos saltan a la vista: primero, la falta de profesionales rigurosamente formados, que ocuparan un terreno delimitado hacia fuera, hacia las demás disciplinas, y definido hacia dentro por la especificidad de sus problemas y sus métodos; $\mathrm{y}$, segundo, la preocupación generalizada por hacer de la escuela una palanca desde la que se trataría de impulsar una idea del país y promover una regeneración social que las minorías sentían como algo necesario para modernizar la nación en el marco de las demás naciones occidentales.

La educación pareció a muchos una 'via regia' para sobreponerse a la crisis social que a lo largo del siglo XIX se fue intensificando, hasta llegar al tiempo crítico del 98. Por ello mismo, estuvo muy pronto ligada a dimensiones ideológicas y políticas que condicionaron su desarrollo.

Tres líneas, en síntesis, podrían servir para ordenar el campo que se abre ante nuestros ojos. Una, que llamaríamos 'central', la que hubo de aproximarse al estudio de las cuestiones de educación y aprendizaje desde un punto de vista científico y positivo, tratando de estudiarlas como procesos reales, sometidos a leyes y analizables con rigor y objetividad. El movimiento krausista, pronto convertido en krauso-positivista, representa esta opción con bastante coherencia y propiedad.

Por la derecha y por la izquierda de la anterior, vendrían a discurrir desarrollos que de algún modo subordinaban la 'escuela' al cumplimiento de unos principios ideológicos que se sentían como superiores y prioritarios. En la derecha mencionada cabe situar la concepción religiosa conservadora, partidaria de una escuela confesional católica; por la izquierda, discurrirá una línea de pensamiento que impone el laicismo racionalista: de ello puede ser buen ejemplo el modelo de la Escuela Moderna que defendiera Ferrer Guardia.

Procuraré, en lo que sigue, reflejar resumidamente el campo que acabo de esquematizar, y trataré de mostrar las tensiones que mediaron la evolución histórica de la disciplina en esos años previos a la guerra civil, en que se fue configurando nuestra primera psicología de intervención.

\section{La primera tradición de psicología escolar. Krausismo e Institución}

El interés por la escuela está ya presente en nues- 
tros humanistas. Luis Vives (1492-1540) ha dejado en sus Diálogos páginas vivas, atractivas, que reflejan la realidad escolar del siglo XVI. Incluso pensó ya, en su Tratado de la enseñanza, en la conveniencia de examinar las habilidades y capacidades del escolar que empieza para dar mayor información y precisión a la labor que con él ha de realizar el educador. Esa idea del 'examen del ingenio' del escolar se convertiría luego en el eje de la obra maestra de Juan Huarte de San Juan (c.1529- c.1588), Examen de ingenio para las ciencias (1575), primera formulación amplia y detallada del proceso de diagnóstico de aptitudes encaminado hacia una orientación profesional (Iriarte, 1939). Pero con todo el interés que ello pueda tener, nos han de ocupar aquí sólo aquellos trabajos cuyo punto de partida viene dado por la nueva psicología científica, que desde fines del siglo XIX estableció un campo nuevo de investigación positiva acerca de la mente humana, sus aptitudes y capacidades, y abrió el camino hacia toda suerte de intervenciones técnicas fundadas en aquel saber.

En 1876, recién estrenado el reinado de Alfonso XII, cobra nueva fuerza un antiguo conflicto entre dos mentalidades con fuerte arraigo en nuestra sociedad: la que propugnaba una visión naturalista, racionalista, positiva, de la realidad, y aquella otra fuertemente conservadora, preocupada por el rigor y la ortodoxia de unas creencias religiosas católicas, y por ello mismo, recelosa de la modernidad que sentía como amenaza a sus principios. El espíritu racionalista había tenido algunas horas altas durante los breves días de la I República (1873). Por entonces se difundieron por el país el krausismo, el evolucionismo, el positivismo, el monismo de Haeckel, el materialismo, y también el anarquismo, y el socialismo (Ruiz, 1975). La restauración dio nuevas alas al mundo conservador. Uno de sus principales exponentes, Juan Manuel Ortí y Lara (1826-1904), reunió en un pequeño volumen, El catecismo de los textos vivos (1884), una serie de violentos ataques a ciertos profesores afines a las nuevas corrientes de pensamiento; lograron, además, que el gobierno impusiera la ortodoxia religiosa en todas las aulas estatales. El choque era inevitable; el resultado fue el abandono de las instituciones oficiales por una serie de educadores e investigadores, convencidos partidarios de la libertad de cátedra y de conciencia. Algunos decidieron crear, en Madrid, una nueva institución educativa, la Institución Libre de Enseñanza, bajo la dirección de Francisco Giner de los Ríos, atenta a los valores de la libertad y de la razón y la ciencia (Luzuriaga, 1957).

El artículo 15 de sus Estatutos se redactó así: "La Institución Libre de Enseñanza es completamente ajena a todo espíritu e interés de comunidad religiosa, escuela filosófica o partido político; proclamando tan solo el principio de la libertad e inviolabilidad de la ciencia, y de la consiguiente independencia de su indagación y exposición respecto de cualquier otra autoridad que la propia conciencia del Profesor, único responsable de sus doctrinas". (Luzuriaga, 1957, 59).

Cuando abrieron sus aulas y pusieron en pié ese centro educativo que iba a ser alma de la renovación modernizadora del país, lo hicieron contando con los nuevos saberes sobre la educación que la recién creada psicología, y la moderna pedagogía, habían aportado.

Giner (1839-1915) era discípulo del introductor de la filosofía krausista en España, Julián Sanz del Rio, y participaba de sus convicciones doctrinales. Concebía el hombre como unidad psicofísica, que por un lado se abría a un fundamento absoluto, y por el otro al mundo de la naturaleza y de la convivencia humana. La dimensión primera era de carácter metafísico, la segunda se desplegaba a través de la ciencia y la moral. Por tener aquella estructura, era la nueva psicología fisiológica una vía básica para comprender al hombre, sobre la que había que fundar la educación. En esa dirección se orienta su psicología, compendiada en sus Lecciones sumarias de psicología $(1874,1877)$ (Lafuente, 1982; Carpintero, 2004).

En la Institución, y en la revista que allí se fundó, el Boletín de la Institución Libre de Enseñanza, (1877-1936), se prestó gran atención a los temas psicológicos relativos a la educación (Herrero, 1999). Los nombres de Binet, Dewey, Claparède, Stanley 
Hall, entre otros, aparecen publicando trabajos en sus páginas.

No obstante, recogeré aquí dos testimonios del círculo krausista, no tan conocidos, y muy directamente ligados a la especialidad que nos ocupa. Me refiero a una reflexión doctrinal, de un catedrático muy notable, Urbano Gonzalez Serrano, y a los estudios sobre fatiga escolar, que analizó Luis Simarro, en uno de sus trabajos juveniles, cuando dirigía el Laboratorio del Museo Pedagógico Nacional.

González Serrano (1848-1904), catedrático del Instituto San Isidro de Madrid, fue discípulo y colaborador de Nicolás Salmerón, el catedrático de metafísica de la universidad de Madrid que por unos meses fue presidente de la Primera República. Don Urbano publicó manuales de sus materias de enseñanza, y múltiples libros de filosofía y psicología; tuvo a su cargo la redacción de la mayor parte de los artículos psicológicos en el Diccionario Enciclopédico Hispano-Americano (1887-1898), obra grande en tamaño y grande en calidad (Montañés, 1989; Jiménez, 1996).

En 1895 publicó un curioso libro, escrito en colaboración con una pedagoga destacada, también próxima a la Institución, y muy sensibilizada hacia el problema feminista, Concepción Saiz y Otero (1851-1934), con el titulo de Cartas... ¿pedagógicas? (Ensayos de psicología pedagógica). Ambos autores mantienen una correspondencia sobre el tema que el título enuncia. Son cartas de personas preocupadas por la educación, y conocedoras de las ideas nuevas.

La pedagoga formula su idea del psicólogo con claridad: "que el psicólogo sea el maestro del Maestro, único medio de obtener educación racional”. Y añade: "no pretendo yo que el Maestro haga Psicología...que eso es cosa de otros, sino que la aprenda para aplicarla constantemente" (Saiz y Otero y González Serrano 1895,104).

Ambos son en cierto modo personalistas, y coinciden en ver a la persona como una realidad dinámica. Para Gonzalez Serrano eso quiere decir que la educación no puede ser un proceso que vaya de fuera adentro, cosa que solo daría 'instrucción', sino algo que excite, impulse y dirija la acción, en una intervención que habrá de ir 'de dentro afuera', con el apoyo del educador. La educación habrá de "provocar el desarrollo mental involutivamente, por intususpección, de dentro hacia fuera" (Id., 128). No se trata de dejar a la imaginación y la fantasía un juego libre, sino de hacer que el espíritu se enfrente con la realidad y se acomode a ella. "El dato real o sugerido es la ocasión y el estímulo para adiestrar la mente" (Id., 131). Habrá que contar primero con la atención general y común, - lo que ambos autores llamarán mirar, la receptividad -, para que la mente de cada cual se module por la perspectiva individual - lo que denominan ver, la espontaneidad creativa de cada cual (Id., 133). Y como ha de contarse con ese fondo dinámico que late en cada individuo al cual hay que atraer hacia los desarrollos enriquecedores, habrá que ver la educación como un sistema de "sugestiones coordenadas y razonadas" (Id., 115), donde la sugestión aparece como el gran medio para dirigir a una espontaneidad ya puesta en marcha (Id., 328).

Para estos dos educadores, el mundo emocional es el fondo primero sobre el que se ha de operar. Gonzalez Serrano se pregunta: "Si la vida emocional es la que propiamente condensa y sazona la savia y frutos de toda educación, pues la inteligencia se limita a instruir o guiar, ¿cómo podemos...educar desde ésta nuestra sensibilidad?". La respuesta viene de inmediato: será gracias al "interés... (que) tiene ...su más firme asiento en la sensibilidad" (Id., 139). Así se llega a decir que "la única fuerza incontrastable es la de la idea, pero la de la idea que arraiga en lo emocional" (Id., 282).

A través de estas cartas construyen un sistema pedagógico convergente con el propio de la Escuela Nueva, centrado en el interés y la sugestión, pero orientado a lograr una eficaz adaptación al medio, (Id., 164). Por ello, les preocupó la idea de crear una 'Pedagogía nacional', arraigada y ajustada a los condicionamientos colectivos que forman el factor social de la personalidad (Id., 239). Anticipando ideas que luego Ortega desarrolló ampliamente en su filosofía, estos educadores fueron conscientes de 
la dimensión 'perspectiva' que tiene toda formación personal, y con ello, la necesidad de "individualizar la educación" (Id., 268).

Emocionalidad antes que intelectualismo, atención, motivación, interés, personalidad, individualidad, socialización, son las variables a las que se presta relieve en esta correspondencia. En suma, se trata de crear una psicología al servicio del maestro, y un psicólogo en apoyo del educador. Tales eran las ideas que vendrían a fijar la relación entre ambos campos disciplinares, Pedagogía y Psicología, en forma muy próxima a la que posteriormente iba a consolidarse de modo muy general.

Con todo, algo más podía hacer el psicólogo en relación con el educador. Luis Simarro (1851-1921), primer catedrático de psicología experimental que ha habido en nuestra universidad, y psiquiatra muy notable, fue durante unos años director de un Laboratorio de Antropología y Psicología Experimental, establecido en el marco del Museo Pedagógico Nacional (Quintana y cols., 2008,46).

El museo se creó en 1882, por inspiración directa de los educadores de la Institución Libre de Enseñanza, como un organismo de apoyo a los educadores, que iba a albergar una biblioteca especializada, y unos laboratorios donde dar cabida a estudios propios de la Pedagogía Experimental. Se encargó de su dirección a Manuel B. Cossío (18581935), y en sus aulas se organizaron conferencias y seminarios que impulsaron la renovación educativa que el país necesitaba (García del Dujo, 1985).

En 1893 Simarro se hizo cargo del mencionado laboratorio, y al parecer, allí se ocupó de temas relacionados con la práctica educativa, especialmente con los problemas de la fatiga escolar. Esta era, sin duda, una cuestión relevante, en un tiempo en que las cuestiones del trabajo y la fatiga en el empleo empezaban a preocupar a fisiólogos e ingenieros, y se cuestionaba el modo como disponer y planificar la tarea en los centros de trabajo. Y la escuela, desde esta perspectiva, no dejaba de ser uno de ellos.

Simarro se hallaba fuertemente vinculado a la Institución. Fue en ella profesor de física, enseñó fisiología del sistema nervioso, dio conferencias, y se preocupó de consolidar el proyecto cultural que veía como vía básica para reformar el país desde la educación.

Aunque su obra escrita es mínima, y su personalidad parece haberla desbordado sin reservas, a juzgar por los juicios de sus contemporáneos, contamos con un trabajo dedicado precisamente a exponer sus ideas acerca de la fatiga y el trabajo escolar. Se trata de su conferencia sobre "El exceso de trabajo mental en la enseñanza" (Simarro, 1889).

A nuestro autor le preocupa el proceso fisiológico y psicológico de la fatiga, y las alteraciones sufridas por los individuos a consecuencia del exceso de trabajo. Se trata de lo que, por entonces, se comenzó a conocer por el gran público con el término francés de surmenage. Los obreros, en sus empresas y fábricas, los administrativos y empleados de oficina, usualmente designados como White collar workers (trabajadores de cuello blanco), y los escolares, atrajeron el interés de médicos y especialistas ocupados en temas de higiene laboral. En 1891 un fisiólogo italiano, Angelo Mosso, publicó un importante estudio sobre fatiga, su medida y sus consecuencias. Por las mismas fechas, algunos otros médicos e investigadores se interesaron por la fatiga escolar, y los modos de hacerla frente. El trabajo de Simarro, pues, se sitúa dentro de una primera oleada de estudios que consideraron el tema de modo teórico, no experimental.

Es fácil advertir en estas páginas el trasfondo evolucionista que las inspira. Se supone en ellas que los esfuerzos mentales, exigidos por el veloz avance de la cultura, consumen en demasía las energías orgánicas. Por ello desde la fatiga, por una escala de grados inexorable, se asciende hacia" la neurastenia, el agotamiento y la degeneración" (Id., 39). Por esa misma razón, dice, degeneran las aristocracias, y se suceden unas etapas de decadencia a otras de esplendor. La sociedad sería "un ser superior", y el individuo se ha de subordinar a ella, pero al precio de un esfuerzo que le termina por agotar mentalmente y le lleva a la degeneración biológica. (Id., 39).

El ensayo sitúa el tema en un nivel propio de los estudios sobre el sistema nervioso. Recuerda las 
ideas de Wundt, quien en su Tratado de Psicología Fisiológica ya veía que con la excitación se ponen en marcha tanto procesos de irritación como de inhibición (Wundt, 1886, cap. vi). Hace luego notar que la fatiga opera disminuyendo la capacidad de funcionamiento, al modo como lo hace también una intoxicación, mientras que la regeneración de su potencia es consecuencia del descanso y, sobre todo, de la nutrición de las células. "La fatiga y la neurastenia,- dice- son comparables a envenenamientos" (Id. 372) y, de ese modo, la fatiga puede ser considerada como un "estado degenerativo", de los que había ya hablado el psiquiatra francés Morel - maestro de Magnan, el maestro parisino de Simarro, no se olvide-. A la inversa, "la degeneración es una especie de fatiga organizada, que ha causado estado, creando una segunda naturaleza" (Id., 371). Tampoco deja de notar que tanto el ritmo de trabajo, como las condiciones de higiene y alimentación afectan al estado del organismo, como también lo hace la condición social de los individuos. Tanto el obrero como el que llama "proletario de levita" y, por supuesto, el estudiante pobre que tiene que trabajar para pagarse sus estudios y se ve forzado a una sobretasa de trabajo, son todos ellos organismos amenazados de tensión y degeneración, que en ocasiones se ven impulsados a refugiarse en la holganza y el juego, abandonando las exigencias de trabajo que demanda la sociedad. Es esta una reflexión en la que se refleja, como en silueta vista al trasluz, la experiencia juvenil del propio Simarro, que hubo de costearse en Valencia sus propios estudios con lecciones y trabajos, y conoció la adversidad desde dentro. Aquí no hay ningún romanticismo: la pobreza, la malnutrición y la sobrecarga de esfuerzo conducen a la degeneración corporal, más o menos transitoria; no hay que dorar la imagen del trabajador explotado. Los datos de la biología permiten formular una teoría que "a su vez da fundamento y legitima las aspiraciones socialistas" en relación a las reformas sociales (Id., 371) que buscan poner fin a toda suerte de explotación del trabajador. En estas reflexiones transparece el espíritu crítico y reformista que alentó en Simarro desde su primera juventud.

\section{La construcción de una Psicología Educativa desde la Paidología}

No bastaba con reflexiones puntuales, fueran teóricas o empíricas. Había que empezar a construir el campo disciplinar. Los maestros habían de aprender las líneas básicas de una psicología que estuviera referida al proceso activo de la educación. Y haría falta disponer de manuales, de libros guía, de información, en una palabra, que permitiera soportar el peso de esas enseñanzas especializadas.

Una de las contribuciones más interesantes fue sin duda la aportación de un hombre ligado a la ILE, y especialmente vinculado al Museo Pedagógico Nacional, que llegaría a dirigir: Domingo Barnés (1879-1940). Se formó al lado de Giner de los Rios y, sobre todo, de Manuel B. Cossío, a quien desde muy temprano ayudaría en el Museo. Pronto tuvo el encargo de enseñar una disciplina dedicada al estudio del niño, la Paidología (Carda y Carpintero, 1993). La Paidología aspiraba a ser una nueva ciencia, en la que se trataba de integrar la psicología con la pediatría y la biología, para así ofrecer de manera unificada una visión científica e integral del niño, en su desarrollo tanto mental como corporal. A Barnés se le deben una serie de estudios en que progresivamente fue ofreciendo un desarrollo cada vez más completo de aquella disciplina (La Paidología, 1932). Introdujo con fuerza la psicología en el curriculum de los maestros, al haber hecho de la paidología su campo de especialización, y lograr luego que esa disciplina se incorporara a los planes de estudio de las Escuelas Normales donde aquellos se formaban, a semejanza de lo ocurrido en otros países, Aspiraba a dar una formación multidimensional a maestros y educadores, proporcionándoles conocimientos rigurosos acerca del niño, sujeto de la educación.

En trabajos sucesivos fue elaborando una visión integradora de ese campo. Para ello previamente realizó una gran labor de registro y acumulación de documentos e información especializada. Esto dio como resultado un volumen, Fuentes para el estudio de la paidología, que publicó el Museo Pedagógico 
Nacional en 1917. Tomó como base documental las publicaciones existentes en el Museo, y pretendía, según su propia declaración, "acercar... al público" las fuentes que allí se encontraban, para con ello "promover así el cultivo de los estudios del niño" (Barnés, 1917, vi).

Había una preocupación educativa en la raíz de este esfuerzo. El autor tenía conciencia de que el maestro necesita de la psicología y de la paidología, porque le resultan imprescindibles para conocer de manera científica al niño, objeto de sus preocupaciones y desvelos. "El maestro que no sepa observar al niño estará incapacitado para hacer del proceso educativo una labor viva, oportuna, adaptada a las exigencias del espíritu del niño, en cada momento de ese proceso..." (Id., 1917, lix). En otros términos, su acción ha de ajustarse al desarrollo de la entidad dinámica que es el alumno. La alternativa posible, e indeseable, habría sido el puro atenimiento a rutinas, la repetición de fórmulas generales, carentes de concatenación con las peculiaridades del sujeto. La psicología y la paidología, concebidas científicamente, son las bases que permiten que la educación se lleve a cabo de un modo personalizado y liberado de las fórmulas vacías.

Esa convicción le lleva a animar al maestro a que se atenga a la observación y al juicio experto de los que han llegado a ser grandes conocedores de la infancia.

En su libro sobre Fuentes organizó el material distribuyéndolo en tres grandes categorías: Fuentes bibliográficas, Instituciones y Congresos. Desde mediados del siglo XIX hasta los días de su publicación, hay datos relativos a los principales países en que florecieron los estudios paidológicos: Alemania, Bélgica, Estados Unidos, Francia, Inglaterra, Italia, Suiza. Lo enriqueció, además, con un índice analítico, cuyos apartados dan ya una primera idea de la concepción paidológica que desde aquí se promovía: "Problemas generales. Higiene escolar. Antropometría. Fisiología. Psicología normal. Psicología patológica. Pedagogía normal. Pedagogía de anormales. Pedagogía social“.

Resulta aquí evidente que la nueva disciplina reu- nía elementos propios de la Psicología, de la Pedagogía, de la Medicina y la Antropología; con ellos quería ordenar y estructurar un conocimiento acerca de la vida y los problemas de la infancia que estuviera dotado de unidad, sistematismo e independencia. Facilitaba, además, bibliografía especializada que podía obtenerse de las colecciones documentales del Museo. El esfuerzo realizado por su autor es importante. Deseoso de que la nueva disciplina arraigara aquí, concibió sin duda la idea de familiarizar a sus nuevos cultivadores con toda aquella información reciente, todavía en circulación, que haría posible no sólo un conocimiento de la historia de la nueva ciencia, sino también de muchos de los trabajos gracias a los cuales se había constituido como un nuevo campo de investigación. Hallamos una confesión notable de propósitos en sus páginas iniciales: "Si en nuestra patria las fuentes bibliográficas fueran más abundantes, y las instituciones fueran más numerosas, y sobre todo, si el manejo de las unas y el conocimiento de la actividad de las otras fueran más asequibles, este libro no tendría razón de ser" (Barnés, 1917, vi). En una palabra, si hubiera efectiva vida científica, este conocimiento estaría ya dado por supuesto. Pero no la hay, y lograr tener un sencillo mapa del campo intelectual, con breves resúmenes de muchas publicaciones recientes, fue la tarea que quiso ofrecer a los educadores españoles, al tiempo que con ello aireaba los recursos y mostraba las posibilidades que el Museo Pedagógico Nacional ponía al servicio de los investigadores.

Desde una concepción moderna de la ciencia, que la veía vinculada con la investigación, y ésta a su vez presente y accesible en el cuerpo de publicaciones que la recogían, Barnés hizo lo posible para proporcionar al nuevo campo sobre el que había de basarse la acción del maestro unas bases conceptuales y documentales a la altura de su tiempo.

\section{Viqueira y la Psicología Educativa}

Lentamente se fue dejando sentir la conveniencia de organizar el campo dentro del marco definido de 
un manual, siquiera fuera breve. Posiblemente el volumen que reúne una información a la vez actual y personal es el que publicó Juan Vicente Viqueira (1886-1924), otra de las figuras notables del círculo de la Institución Libre de Enseñanza.

Formó parte del núcleo más cercano a Francisco Giner y Manuel B. Cossío, teniendo relación de parentesco con éste último. Con hondas raíces en Galicia, fue catedrático de instituto de filosofía. Padeció desde muy joven una grave enfermedad que tuvo muy temprano fatal desenlace. Pero a pesar de ello, se interesó por la filosofía y la psicología, y con la ayuda de la Junta para la Ampliación de Estudios (JAE) viajó y estudió con maestros como Edmund Husserl, Henri Bergson, Karl Sumpf o George Elias Müller, y llegó a trabajar un tiempo con éste último en Gottinga, resultando de ello un artículo de carácter experimental sobre procesos de memoria que apareció en Psychologische Forschung, una publicación alemana muy respetada. En suma, podría haber sido el primer psicólogo español de alcance internacional, si hubiera podido tener más larga vida, y una obra más cumplida.

Trazó las líneas elementales de una psicología educativa en su Introducción a la Psicología Pedagógica (1919), un pequeño manual que ofrece los conceptos básicos de los procesos psicológicos que desde la infancia operan para terminar constituyendo una personalidad integrada. Allí deja claro que el maestro necesita conocer tales conceptos para cumplir eficazmente con su misión."Si educar es intervenir conscientemente en la evolución del individuo, es preciso conocer este individuo, su evolución y poseer los métodos diagnósticos de esta evolución" (Viqueira, 1919, 20-1).

No carece de interés el ordenamiento general que propone dar a la formación psicológica que debe dársele al maestro. En su opinión, éste debería conocer:

“A) La Psicología General o la organización del espíritu en general. B) La evolución mental en general (también Psicología general). C) Los diferentes tipos de organización y de evolución (Psicología individual). D) Los medios diagnósticos y técnicos, de aplicación, de la Psicología (Psicología aplicada o Psicotecnia) "(Viqueira, 1919, 21).

Como se ve, se trata de una formación teórica que está directamente orientada a la intervención en el proceso educativo, y que tiene en cuenta los aspectos propios de las diferencias individuales, y las particularidades de los métodos de intervención.

Construye su texto desde una base evolucionista, y al tiempo demanda una integración de la descripción fenomenológica con la explicación naturalista y experimental, desde una afirmación de la conciencia como horizonte de la psicología: "La psicología se ocupa de la conciencia y ha de tener en cuenta sus condiciones naturales" (Viqueira, 1919, 7). Se sitúa, así, en la línea en que se habían venido moviendo su maestro G.E. Müller, y antes que él, el propio William James. Para él, los dos grandes tipos de procesos son los intelectuales y los afectivos, cuya síntesis determina precisamente la organización final de la personalidad individual.

La conciencia representa la forma personal de la experiencia, aquella 'vida interior' del sujeto que le abre justamente al mundo exterior, al tiempo que se producen en el sistema nervioso determinados procesos. La relación entre esas dos series de fenómenos la concibe dentro del modelo del paralelismo, siguiendo en esto las enseñanzas de W. Wundt.

En su desarrollo didáctico, presta mucha atención a aspectos sensoriales, lenguaje, desarrollo del espacio, del dibujo y del juego. Y el libro se cierra con una bibliografía especializada, particularmente detallada, y en que se reúnen autores como Wundt, Ebbinghaus, Binet, Stern, Baldwin, Meumann y muchos más.

Viqueira creía que este campo merecía que se realizara un importante esfuerzo gubernamental, que hiciera posible la formación psicológica de los maestros, y que recogiera de las investigaciones recientes aquellas lecciones y recomendaciones que pudieran mejorar el sistema educativo de nuestras escuelas. Propuso que se creara un Instituto de Psicología de la Educación, en un informe personal que dirigió a la Junta para Ampliación de Estudios e Investigaciones, como conclusión de su estancia en 
Alemania (Viqueira, 1915). Allí había podido ver las ventajas indiscutibles que reportaba al sistema educativo la existencia de una interacción y una permeabilidad que hacían posibles la transferencia de lo investigado en el laboratorio a la acción educativa llevada a cabo en el aula. Su informe no tuvo consecuencias; sus ideas quedaron encerradas en las páginas de su escrito, que fue un informe más de los que los becarios de la JAE enviaban a ésta. La semilla de una psicología educativa aplicada quedó sin fructificar.

\section{Las aportaciones maduras. Lafora y la Psicopatología.}

Tal vez el intento más logrado de penetrar la ciencia psicológica en el mundo del magisterio en los años anteriores a la guerra civil, haya sido el representado por la obra de Gonzalo Rodriguez Lafora (1886-1971).

Fue una gran figura de la psiquiatría de la primera mitad del siglo XX. Dedicó considerable atención a los aspectos psicopatológicos de los niños, y creyó imprescindible dar una información rigurosa y actual de los principales síndromes psicopatológicos a los maestros, para lograr así una mejora sustancial en la salud mental de las escuelas.

Había adquirido una formación muy rica y rigurosa en Alemania primero, con Kraepelin, Alzheimer, Ziehen y otras grandes personalidades de la clínica psiquiátrica germánica, y luego en Estados Unidos, donde amplió sus conocimientos sobre neurología al lado de Shepherd I. Franz, el gran investigador de la UCLA sobre las bases neurales del aprendizaje quien, como su colega Lashley, estudiaba experimentalmente los efectos que la ablación progresiva de tejido cerebral tenía sobre los aprendizajes previos; en estas investigaciones colaboró activamente el discípulo español.

Sin duda sus maestros le hicieron ver la necesidad de poner al día a nuestros educadores, sobre aquellas cuestiones de tipo clínico que aparecían en la escue- la y afectaban al niño y a su educación. En 1917, publicó Los niños mentalmente anormales (Rodríguez Lafora, 1917), libro "principalmente dedicado a los maestros" (id., xii), porque, como en el principio del texto se declara, "el problema de los niños mentalmente anormales se presenta con toda su pujanza en aquellos países progresivos en que la asistencia a la escuela es obligatoria" (Id., 1). Los trastornos psicológicos tienen una inmediata plasmación en las perturbaciones que experimenta el proceso educativo de aquellos escolares que los padecen.

En realidad, sus preocupaciones giraban en torno a dos cuestiones básicas, estrechamente relacionadas entre sí: el diagnóstico de las deficiencias, y la pedagogía especial, que busca introducir los complementos terapéuticos que pueden poner un remedio más o menos eficaz a la situación dada.

La perspectiva psicológica aquí enlaza con la más puramente médica, y con la propia de la pedagogía, dado que las tres disciplinas tienen mucho que ver y que decir ante los problemas mentales de los escolares. Recomendó, por ello, establecer tres exámenes complementarios entre sí, cada uno dedicado a cada una de las mencionadas disciplinas, aunque era plenamente consciente de que los déficits y las patologías afectaban en definitiva a la personalidad integral del niño, asumiendo así el punto de vista holista de la psicología individual.

No se conformó con escribir el libro. También fundó en 1925 un Instituto Médico-Pedagógico en Madrid (Carabanchel), al tiempo que inauguraba un Sanatorio Neuropático. En el primero, contó pronto con la colaboración de dos personas que iban a poner en marcha la psicología rigurosa, científica, aplicada al mundo de la clínica escolar, el médico y psicólogo José Germain (1897-1986), y la maestra y psicóloga Mercedes Rodrigo (1891-1982), ya formada en Ginebra al lado de J. Piaget y E. Claparède. Ambos, trabajando en el mencionado instituto, dieron cima a la obra Pruebas de Inteligencia, la primera edición española rigurosa y baremada del test de Binet en la versión revisada de Terman (Germain y Rodrigo, 1930). Allí tuvo una de sus raíces la psicología contemporánea española. 
Lafora mantuvo vivo su interés por la clínica psicopedagógica largo tiempo. En los años treinta, cuando la pedagogía llegó al fin al ámbito de la universidad, y se organizaron sus estudios, en la que iba a ser la Facultad de Filosofía y Letras del decano García Morente. Esta fue, como es bien sabido, el gran centro universitario de extraordinaria calidad en que enseñaron Ortega y Gasset, Zubiri, Menéndez Pidal, Américo Castro, Claudio Sánchez Albornoz, Asín Palacios y tantos otros nombres de máxima valia de la vida intelectual de la España contemporánea. En ella alcanzaron por primera vez los estudios pedagógicos el nivel universitario. Precisamente, Lafora hizo acto de presencia en aquellos estudios, y allí enseñó una Psicopatología Infantil y Pedagogía de Anormales Mentales, si bien no era éste uno de los cursos centrales. La amistad que le unía a Ortega y Gasset, así como su personal valía y el prestigio que como psiquiatra tenía, y que le llevó a presidir el Consejo Superior Psiquiátrico en aquellos años, explican sin duda su presencia como docente de aquella singular Facultad (Carpintero, 2008).

\section{Escuela e ideología}

La sociedad española vivió durante el siglo XIX un movimiento de renovación y regeneración, que buscó poner al día un país que se había distanciado de la modernidad durante la Edad Moderna, precisamente como resultado de una opción ideológica básica, la defensa de la ortodoxia católica y el rechazo del libre pensamiento, desde los días de la reforma luterana.

Este hondo problema vino a introducir una polarización en la convivencia, de manera que progresistas y conservadores multiplicaron sus discrepancias al tratar de resolver las cuestiones básicas de la convivencia - la idea del hombre, la actitud ante la razón y la ciencia, la modernidad tecnológica, la religión, el gobierno, la idea nacional, y, por lo que aquí respecta, la educación. Crecientemente se fueron configurando dos grandes sectores sociales - las "dos
Españas" (Figueiredo, 1933) - muy conservador uno y muy progresista el otro, que envueltos en una espiral de tensión terminaron por enfrentarse violentamente en la guerra civil de 1936-39. Ambos estuvieron sin duda condicionados por muchos factores; pero sin duda uno muy determinante fue el de la educación.

Así, mientras se levantaba el frágil edificio de una educación basada en los nuevos conocimientos de la ciencia, la Psicología y las nuevas técnicas educativas, fue también creciendo la tensión entre una escuela fuertemente religiosa y conservadora, vehículo transmisor de una concepción del mundo que contaba con el apoyo de la Iglesia Católica y otra de orientación laicista, que aspiraba a implantar en el país los principios del librepensamiento y el racionalismo ideológico.

Ejemplos de orientación religiosa fueron, entre otras, las escuelas que fundó en Granada el P. Andrés Manjón (1846-1923), catedrático de la facultad de Derecho de aquella universidad, sacerdote y educador. Movido por la contemplación de su circunstancia inmediata, se convirtió en promotor de una enseñanza cercana a la naturaleza, fundada en las doctrinas y principios de la Iglesia católica y su moral, e inspirada en principios de la enseñanza activa guiada por el interés del niño. El movimiento de estas escuelas, llamadas "del Ave María”, buscó primero atender a los niños del Sacro Monte granadino, pero se extendió pronto por el país sin dificultad.

Hay también algunos otros trabajos que buscaron combinar la psicología científica y la cosmovisión religiosa, como es el caso del Curso de psicología escolar para maestros (1916) que publicó un religioso capuchino, el P. Francisco de Barbens, (18751920). Quería superar lo que consideraba que era "nuestro retraso pedagógico", y para ello aspiraba a formar al maestro en el conocimiento de una psicología que sirviera de base a la educación, y que a su vez estuviera fundada en datos "de histología cerebral, de fisiología, de química fisiológica, de psicología normal y de psiquiatría" (Barbens, 1916, v-vi).

Semejantes fundamentos requieren del maestro 
un conocimiento adecuado, que le capacite para la acción educadora sobre la personalidad infantil. Aunque el autor es profundamente espiritualista, asume plenamente la existencia de unos condicionamientos orgánicos, nerviosos y químicos, de la actividad cerebral que hace posibles los procesos mentales. Da importancia a la nutrición, al deporte, al juego, y aprecia el papel de la medición en la Psicología y la educación. Incluso recoge los ítems del test de Binet-Simon, para información de los maestros que han de leer el libro (Barbens, 1916, 60-63). Hace del concepto de aptitud un tema central, y estima que el conocimiento de las que enriquecen a los distintos individuos, ha de servir de base al proceso educativo; es, pues, una doctrina que se presenta como "psicología individual". Para él son claves de la educación el interés, la memoria y los sentimientos, que modulan la inteligencia. Reconoce la parte de verdad que, a su juicio, tienen tanto la afirmación de los sentimientos que hiciera Ribot, como la de las ideas-fuerza que defendió Fouillée (Id., 217), una vez conciliadas ambas y eliminadas sus extremosidades.

Hay en su libro aspectos modernos junto a otros claramente regresivos. Valga como ejemplo de los primeros su reconocimiento de los distintos tipos de escuela o de enseñanza: la basada en la obediencia (escuela 'latina') y la dominada por la libertad (escuela 'anglosajona'); entre ambas situaría una tercera inspirada en los principios educativos de M.Montessori (Id., 326 ss). Como se ve, los estilos educativos están aquí relacionados con variedades culturales básicas, o lo que él llama "las leyes de la raza" (Id., 326). En cambio, mantiene una posición cerrada frente al tema de la coeducación, a la que atribuye un pernicioso influjo sobre las personalidades infantiles; lo llama 'hermafroditismo psíquico', que se produciría porque a la niña "se la instruye, se le despiertan aficiones, se la forma con los mismos procedimientos, estímulos y con el mismo fin con que se forma el alma del niño" (Id., 324). En defensa de la individualidad y diferenciación de género, cree que hay que proscribir ese método.

Tras considerar aspectos relativos a los temas psi- copatológicos, termina por hacer un encendido reconocimiento del magisterio católico, la figura de Jesucristo, y el valor formativo de la religión. Se opone así a lo que llama el 'positivismo pedagógico' (Id., 481), que excluye la religión y todos sus contenidos del ámbito de la educación escolar.

El otro extremo ideológico podría representarlo el movimiento de la Escuela Moderna, liderado por Francisco Ferrer (1859-1909), maestro anarquista y miembro de la masonería, que difundió principalmente en Cataluña los ideales de una escuela laica y basada en un saber empírico y positivo. Su figura ha quedado ligada al proceso de la Semana Trágica de Barcelona, de 1909, con la que las autoridades lo relacionaron como promotor ideológico, y de resultas de lo cual fue condenado a muerte y fusilado en Montjuich (Barcelona).

Ferrer había tenido una formación francesa, de orientación positivista, y una inclinación fuerte hacia las posiciones políticas radicales. Terminó por pensar que la reforma del país había de pasar por la educación, y dedicó a ello sus esfuerzos de madurez, a la postre frustrado por el mencionado movimiento revolucionario fracasado.

Resumen bien el espíritu de su proyecto unas breves líneas introductorias de su programa: "La misión de la Escuela Moderna consiste en hacer que los niños y las niñas que se le confíen lleguen a ser personas instruidas, verídicas, justas y libres de todo prejuicio. Para ello, sustituirá el estudio dogmático por el razonado de las ciencias naturales. Excitará, desarrollará y dirigirá las aptitudes propias de cada alumno, a fin de que con la totalidad del propio valer individual, no sólo sea un miembro útil a la sociedad, sino que, como consecuencia, eleve proporcionalmente el valor de la colectividad. Enseñará los verdaderos deberes sociales... y, principalmente, para realizar el propósito de la Escuela Moderna, encaminado a preparar una humanidad verdaderamente fraternal, sin categoría de sexo ni clases, se aceptarán niños de ambos sexos desde la edad de cinco años" (Ferrer, 1978, 26-7). Desde su comienzo, excluyó la enseñanza de la religión y promovió la coeducación, cuestiones que levantaron los áni- 
mos de muchos grupos conservadores contra esta escuela. Eliminó también los premios, "todo porte de medallas, triángulos y cintajos" (Id., 92), para buscar sólo la persuasión racional como instrumento formativo.

Defensor de la personalidad de cada escolar, llegaría a proponer que "la pedagogía oficial ha de ceder el puesto a la individualidad" (Id.65), e hizo del respeto a la naturaleza el camino esencial de la educación. Algunas de sus semillas terminarían por florecer en la escuela de los años de la Segunda República.

\section{Conclusión}

La modernización del país, tras la crisis del 98, incluyó como uno de sus aspectos básicos, un proceso de crisis y renovación de la educación. Resultaba inevitable que los desarrollos de la nueva Pedagogía y la nueva Psicología, que florecían en los países de nuestro entorno, dejaran su huella sobre los proyectos educativos nacidos en la nueva situación. Sin embargo, la estrecha implicación entre la educación y la concepción del hombre que ha de resultar del proceso educativo hizo que, desde ideologías bien dispares, resultaran fórmulas y planes formativos diferentes.

Ciertos elementos, propios de la época, como la importancia de los conocimientos psicológicos por parte de los educadores, el papel dominante del interés, y la necesidad de una sustitución del verbalismo por la actividad escolar, se asumieron fácilmente desde las más varias posiciones. Pero el núcleo ideológico, relacionado con el papel de la religión o la coeducación de sexos en el aula, se mantuvo rígidamente por los distintos grupos, y con ello, la educación se vio envuelta en los avatares de la vida política del país. Y con la educación, también, por supuesto, la Psicología de la Educación.

El retraso en aparecer la Psicología como profesión, proceso que tendría lugar ya en los años cincuenta del siglo pasado, hizo que las cuestiones de psicología educativa estuvieran largo tiempo en manos de médicos y educadores, interesados por los problemas de un campo que es de suyo profundamente interdisciplinar. No obstante, es posible ver, en este proceso de desarrollo que fue desenvolviéndose en los años que precedieron a la guerra civil, la existencia de una creciente interrelación de los investigadores españoles con los especialistas de centros extranjeros, y la progresiva constitución de los distintos elementos que integran un verdadero campo de especialización profesional.

Deliberadamente nos detenemos en la frontera que, aquí como en tantas otras cosas, marcó el conflicto de la guerra española. Lo que vino después sólo en parte estuvo condicionado por estos antecedentes de preguerra, porque el nivel de destrucción de la situación anterior fue altísimo, y personas e instituciones fueron destruidas o hubieron de exiliarse. Pero estos elementos son sin duda piezas clave que aportan contenidos esenciales para la construcción de la identidad actual de la especialidad.

Los tiempos han cambiado, y las situaciones son otras. La Psicología de la Educación ha conocido desarrollos decisivos en las décadas pasadas. No obstante, la conciencia de las implicaciones sociales y políticas con las que está imbricada es algo que no puede olvidarse, si se quiere hacer una labor que se ajuste a las condiciones del entorno real del país.

\section{Referencias}

Barbens, F. de (1916). Curso de psicología escolar para maestros. Barcelona: Gili.

Barnés, D. (1917). Fuentes para el estudio de la Paidología. Madrid: Museo Pedagógico Nacional.

Barnés, D. (1932). La paidología, 3 ed. Madrid: Espasa Calpe.

Barrio, V. del y Carpintero, H. (1985). Los comienzos de la psicología educativa en España: La tradición krausista, Revista de Historia de la Psicología, 6, 133-143.

Blanco, F. y Rosa, A. (1989). La visión de Viqueira sobre la psicología infantil y pedagógica, Revista 
de Historia de la Psicología, 10, 117-125.

Carda, R. y Carpintero, H. (1993). Domigo Barnés: Psicología y educación. Alicante: Instituto de Cultura Juan Gil Albert.

Carpintero, H. (2004). Historia de la psicología en España. Madrid: Pirámide.

Carpintero, H. (2008). Lucio Gil Fagoaga y los estudios de psicología para filósofos y educadores. En VVAA., La Facultad de Filosofía y Letras de Madrid en la Segunda Republica (pp. 287-293). Madrid: Ministerio de Cultura.

Ferrer, F. (1978). La Escuela Moderna, 3 ed., Madrid: Zero.

Figueiredo, F. de (1933). Las dos Españas. Santiago: Universidad de Santiago de Compostela.

García del Dujo, A. (1985). Museo pedagógico nacional, 1882-1941. Teoría educativa y desarrollo histórico. Salamanca: Instituto de Ciencias de la Educación.

Germain, J. y Rodrigo, M. (1930). Pruebas de inteligencia. Madrid: Espasa Calpe.

Herrero, F. (1999). Educación y regeneración. Una aproximación cuantitativa a través del 'Boletín de la Institución Libre de Enseñanza'. En L. Romero y cols., Cultura y psiquiatría del 98 en España. (pp. 119 y ss). Madrid: Necodisne.

Iriarte, M. de, S.I. (1939). El doctor Huarte de San Juan y su 'Examen de Ingenios. Contribución a la historia de la psicología diferencial, Madrid: Jerarquía.

Jiménez, A. (1996). El krausopositivismo de Urbano Gonzalez Serrano. Badajoz: Diputación Provincial.

Lafuente, E. (1982). La psicología de Giner de los
Rios y sus fundamentos krausistas, Revista de Historia de la Psicología, 3, 247-270.

Luzuriaga, L. (1957). La Institución Libre de Enseñanza y la educación en España. Buenos Aires: Universidad de Buenos Aires.

Mestre, M. V. y Carpintero, H. (1982). Psicólogos españoles: Juan Vicente Viqueira (1886-1924). Revista de Historia de la Psicología, 3, 133-156.

Montañés, J. (1989). Urbano González Serrano y la introducción del positivismo en España. Cáceres: Diputación Provincial.

Ortí y Lara, J. M. (1884). El catecismo de los textos vivos. Madrid: Biblioteca de la Ciencia Cristiana.

Quintana, J. y cols. (2008). El lugar de la psicología en la universidad española del siglo XX. Madrid: Universidad Autónoma de Madrid.

Rodriguez Lafora, G. (1917). Los niños mentalmente anormales. Madrid: La Lectura.

Ruiz, D. (1975). La mentalidad positiva en España: desarrollo y crisis. Madrid: Tucar.

Saiz y Otero, C. y González Serrano, U. (1895). Cartas... ¿pedagógicas? (Ensayos de psicología pedagógica). Madrid: Victoriano Suarez.

Simarro, L. (1889). El exceso de trabajo mental en la enseñanza, Boletín de la Institución Libre de Enseñanza, 13, 37-39, 88-91, 369-373.

Viqueira, J.V. (1915). La enseñanza de la psicología en las Universidades alemanas. Madrid: Junta para Ampliación de Estudios e Investigaciones Científicas.

Viqueira, J.V. (1919). Introducción a la psicología pedagógica. Madrid: Beltrán.

Wundt, W. (1886). Éléments de psychologie physiologique. Paris: Alcan, 2 vols.

Manuscrito recibido: 01/10/2010

Revisión recibida: 03/11/2010

Manuscrito aceptado: 10/11/2010 